\title{
Importance of Cumulus Cells in Oocyte Maturation and Conservation Strategies to Improve Vitrification Results
}

\author{
Fahiel Casillas ${ }^{1 *}$, Alma López², Miguel Betancourt ${ }^{2}$, Socorro Retana-Márquez¹, Lizbeth Juárez-Rojas ${ }^{1}$ \\ ${ }^{1}$ Department of Biology of Reproduction, Mexico \\ ${ }^{2}$ Department of Health Sciences, Mexico
}

*Corresponding author: Fahiel Casillas, Department of Biology of Reproduction, Autonomous Metropolitan University-Iztapalapa Unit, Mexico.

\section{Abstract}

Vitrification is not a recent technique, it was firstly described in 1934, but was not successful until years later because the effects of the cryoprotectants (CPAs) used were unknown. Later, in 1985, Rall and Fahy [1] resumed the application of ultrafast cryopreservation by reducing the toxicity of the media and decreasing exposure times, modifying temperatures in order to improve their results [2]. In this way, the cryopreservation basis has been broadened with the aim of optimising conditions for the development of this process. Some of the main concerns are, the formation of intracellular and extracellular ice crystals, the CPAs toxicity and the osmotic changes during vitrification [3].

The large amount of lipids in porcine oocyte cytoplasm and the presence of the surrounding cumulus cells (CC), which are necessary for the meiotic resumption through gap communicating junctions, have been linked to less CPAs cell permeability and protection leading to cell damage [4]. However, previous studies indicate that the presence of the CC is essential to protect oocytes against damage induced by the vitrification procedure compared to the vitrification of denuded oocytes [5]. Also, it was reported that cumulus-oocyte complexes (COCs) vitrification reduces the viability of the CC while preserving oocyte viability [6]. This is important to be considered since these cells establish communication with the oocyte for the exchange of molecules such as: cAMP, ions, pyruvate, among others, which are required for the acquisition of its mitochondrial function, maturation and fertilizaton capacity. Therefore, maintaining the viability of the CC is a key factor for the vitrification of immature oocytes. Consequently, vitrification of immature porcine oocytes has been more difficult to achieve than other species such as sheep, cattle, hamsters, mice, human, among others [7-10] and this is why it still remains a challenge for cryobiologists.

\section{Introduction}

Vitrification can be used for the cryopreservation of cells, tissues, organs and even organisms. However, its current use is mostly associated to gametes due to its importance in human assisted reproduction techniques and animal production. This technique requires the use of CPAs added to media in high concentrations and devices with high vitrification-warming rates to reduce ice crystals formation. Due to their chemical characteristics, CPAs are highly toxic. If these substances are misused, they can cause cell damage and consequently cell viability loss.
Recently, several studies have been carried out to improve vitrification procedures with the aim of increasing viability and embryo production rates for obtaining live offspring. However, few studies have evaluated the effects on oocytes viability in the germinal vesicle (GV) stage after vitrification. This is because one of the main factors that impact on viability is the nuclear state of the cell. It is known that GV oocytes are more sensitive to cooling compared to those in metaphase II (MII) or embryonic stages [11]. This impact on viability is due to structural changes in the membrane of porcine 
oocytes as the transition temperature of the lipid phase is affected. It is currently known that the cell lipid content varies between species. In porcine the triglyceride content is higher compared to other domestic species. Therefore, due to the high amount of lipids and the presence of the CC, immature porcine oocytes have low CPAs permeability into their intracellular space [4]. Several studies have implemented techniques to reduce the lipid content in porcine, these techniques include mechanical delipidation, polarization of cytoplasmic lipid droplets by centrifugation and partial removal of lipids by micromanipulation $[10,12,13]$. This suggests that the low viability rates are due to insufficient permeability of CPAs.

\section{Oogenesis}

In mammals the oogenesis is produced by mitotic and meiotic divisions. In the first division process the chromosome number is maintained diploid ( $2 \mathrm{n}$ ) but later by meiotic division processes it is reduced to half to produce haploid (n) gametes. Oogonia are diploid cells $(2 n)$ that are produced from the primordial germ cells during embryonic development. Later, these cells undergo meiosi to become primary oocytes. The onset of first meiotic division, in most mammals, is arrested at the stage of diplotene at prophase I during fetal life and resumes until puberty is reached. The nucleus of the oocyte is known as the GV. During puberty, the action of gonadotrophins allows the process of meiotic resumption and extrusion from the first polar body at the stage of MII. Again, the meiosis is stopped until the processes of ovulation and fertilization are generated. If the oocyte is fertilized, the second meiotic division is completed, which includes the expulsion of the second polar body and the formation of the pronuclei. Finally, a zygote is obtained, which again presents a diploid chromosome number (2n) [14].

\section{Oocyte Maturation}

During the oogenesis, the oocytes gradually acquire nuclear and cytoplasmic maturation during their growth. Meiotic competence, which is the ability of the oocytes to resume meiosis and become nuclear mature, is acquired during folliculogenesis and is related to the formation of the antrum, where the oocytes reach approximately $80 \%$ of their final size. The development of the oocyte competence is related to the cytoplasmic maturation and refers to its capacity to be fertilized and develop an embryo capable of continuing its development to term and produce a living individual. Cytoplasmic maturation is acquired after the oocyte is meiotically competent. An oocyte that has acquired meiotic competence does not necessarily acquire cytoplasmic maturation. Meiotic maturation involves a cascade of processes, initiated with a surge of LH, promoting the progress of the oocyte to MII and to the extrusion of the first polar body. After the LH surge, an event that is essential for the resumption of meiosis, is the expansion of the CC, which is caused by the production of hyaluronic acid from these cells in response to gonadotropins [15].

\section{Meiotic Arrest}

Mammalian oocytes, unlike amphibians or echinoderms, have spontaneous maturation when released from the follicles and cultured in vitro. Therefore, it has been proposed that the follicular cells that surround the oocyte, the $\mathrm{CC}$, are responsible for this arrest through the production and release of molecules such as cAMP or purines such as adenine and hypoxanthine. The inhibition of the follicular cells can be transmitted to the oocyte through the follicular fluid, by the communicating junction as it is the case of the mouse and the rat [14]. High levels of cAMP, supplied mainly by the $\mathrm{CC}$, allow the oocyte to be held in prophase I and only released from the inhibitory effect when the cell communication is interrupted, and the cAMP levels drop. This decrease will inactivate cAMPdependent protein kinase (PKA) and through a signaling cascade, a phosphatase is activated that dephosphorylates the maturation promoting factor (MFP) [14].

\section{Mechanism of Meiotic Arrest}

The cAMP produced through the stimulation of the G-protein called Gs by the G-protein coupled receptor 3 (GPR3), is transported to the oocyte by the $\mathrm{CC}$ and/or captured by phosphodiesterase $3 \mathrm{~A}$ inhibitors (PDE3A) in the follicular medium. AMPc-dependent protein kinase regulates MPF activity by CDC25 phosphatase, and Weel/Mytl kinase: cDC25 dephosphorylates cyclin-dependent kinase (CDK1), although Weel/Mytl phosphorylates it. High levels of cAMP result in the phosphorylation of CDK1 and the FPM complex, CDK1/cyclin B (CyB) becomes inactive, so that the oocyte remains arrested in the GV stage [16]. Another model proposed for the detention in prophase I is due to the action of purines, where adenosine stimulates adenylate cyclase through a membrane receptor, while hypoxanthine transferred by follicular cells through the communicating gap junctions prevents the hydrolysis of cAMP, by inhibiting cAMP-phosphodiesterase. The effect is that no resumption of meiosis is generated by the maintenance of high levels of cAMP [14].

\section{Mechanism of Meiotic Resumption}

Gonadotropins bind to their receptors on the CC producing an increase in the production of cAMP. Protein kinase A type II (PKAII) is activated by this increased cAMP in the granulose cells leading to the regulation of CREB in the transcription of the cre-gene, including the transmembrane precursors of epidermal growth factors (EGF), which are converted into mature peptides possibly by the protein kinase C (PKC) pathway. EGF factors bind to their EGFR receptor by activating mitogen-activated protein kinase (MAPK) using phosphatidyl inositol 3-kinase (PI3K)/protein kinase B (PKB) and down-regulation of steroidogenesis. Activation of MAPK and PI3K/ PKB decreases the level of cAMP in the oocyte by phosphorylation of connective $43(\mathrm{Cx}-43)$ and activation of PDE3A, respectively, followed by the FPM complex which is activated for meiotic 
resumption. The accumulation of cGMP by nitric oxide (NO) and/or natriuretic peptides by FSH stimulation serve to prevent premature oocyte maturation, while the decrease of this second message after LH treatment is involved in oocyte maturation and ovulation [16].

\section{Follicular Cells and Oogenesis}

The primordial follicles of mammalian ovaries are made up of an oocyte, detained at the prophase of the first meiotic division, surrounded by a single layer of follicular cells. The follicular cells emit cytoplasmic projections towards the oocyte establishing communicating unions. At the end of the follicular growth period, the oocyte stops growing and remains surrounded by several layers of follicular cells, known from that moment as CC or COCs [14]. Communication between germ cells and somatic cells is necessary for the survival of the oocyte and its development. Low-molecular-weight nutrient substances such as pyruvate and metabolic precursors such as amino acids and nucleotides are transferred through the communicating bonds. These substances make it possible to conclude the first meiotic division and acquire its fertilizing capacity. After an increase in LH levels, which induces ovulation, intercellular signals are interrupted by the production of hyaluronic acid by the CC, which causes its dispersion.

MPF was originally described in amphibian oocytes. It is a widely conserved protein complex from an evolutionary point of view, since it has been found in similar forms from yeasts to humans, both in somatic and germ cells. In mammals it has been found in species such as cattle and pigs. The fundamental role of MPF is to trigger the transition of cells from G2 to M phase in the cell cycle, but because cyclic changes in MPF activation are crucial in the regulation of the mitotic cycle in somatic cells, in oocyte maturation, it may involve similar cyclic changes in MPF activity in oocyte maturation [14].

\section{Hormonal Role of Follicular Cells}

Under the influence of gonadotropins, the follicles synthesize steroid hormones such as androgens and estrogens, which contribute to follicular development by inducing the proliferation and differentiation of granulosa cells through androgen receptors (AR) and estrogen receptors (ER), respectively. Theca cells produce androgens such as androstenedione under the influence of an LH stimulus, while granulose cells produce estrogens such as oestradiol, using androgens as a substrate, under the influence of FSH. Androgens participate in the proliferation and survival of granulosa cells through AR. Female rats with an AR deficiency are subfertile with a reduced number of antral follicles and ovulated oocytes, with a high rate of apoptosis in granulosa cells. Thus, specific AR for granulosa cells are essential for follicular development and survival [17]. Oestradiol is the predominant estrogen in terms of estrogenic activity. One of the main functions of pre-ovulatory granulosa cells is the synthesis of oestradiol. Within the follicle, oestradiol is produced by the enzyme aromatase, increasing the response of granulosa cells to gonadotrophins. Oestradiol plays an essential role in gonadotrophin-induced follicular differentiation. The activins and inhibins produced by granulose cells play essential roles in paracrine functions by regulating the LH-induced synthesis of androgens produced by theca cells, and thus ensure the supply of oestradiol. The activin-inhibin system not only regulates the proliferation of granulose cells, but also their differentiation and oocyte maturation (accelerated by the action of activin A) [17].

\section{Cumulus Cells and Fertilization}

The extracellular matrix of the CC is mainly composed of hyaluronic acid conjugated with various proteins, giving the tissue a resistant structure. At the beginning of meiosis, the matrix is secreted by the CC, achieving their expansion before ovulation. In in vitro conditions, when oocytes are incubated in the presence of FSH and LH, expansion of the CC occurs, indicating that the oocyte has matured [14]. Sperm must be able to penetrate through the CC. Its acrosome contains various enzymes such as hyaluronidase and acrosine, which are acquired during sperm maturation in the epididymis and will be responsible for its ability to penetrate. In some mammal species such as pigs and cattle, the presence of the CC makes IVF more efficient because they increase sperm mobility and promote the acrosome reaction [18].

\section{Co-culture System with Cumulus Cells}

Maintaining the quality of the oocytes is a critical and important aspect of completing the processes of maturation, fertilization, implantation, and embryo development. Studies indicate that removing the CC prior to IVM decreases oocyte quality in species such as mice and pigs $[19,20]$. However, removing the CC is essential for some manipulation techniques such as vitrification of GV oocytes or intracytoplasmic sperm injection (ICSI) [21]. Therefore, the use of a co-culture with CC is intended to restore the development potential of denuded oocytes because studies have reported that these cells have an important role in the maturation process, regulating meiotic progress for nuclear and cytoplasmic maturation [6,22]. For example, reduced glutathione synthesis (GSH) in COCs during IVM has been reported in swine [23]. Studies indicate that the CC have an important role in GSH synthesis, which exerts a cellular protection to decrease oxidative stress. This is important because in vitro cultures are maintained at high concentrations compared to an in vivo environment [24, 25]. This generates an increase in reactive oxygen species leading to a decrease in antioxidant levels and cell death by apoptosis [26].

The beneficial effects of oocytes-CC co-cultures are the secretion of factors such as nutrients, substrates, growth factors and their detoxifying power from the culture media [27]. A study using human oocytes indicated that the use of a co-culture of human oocytes with autologous CC decreased their fragmentation and increased the number of embryos that reached the 4-8 cell and blastocyst stages [28]. 


\section{Conclusion}

Gap junctions and paracrine signalilng constitute the two ways of intracellular comunication between oocytes and CC. In mammals, CC display important roles for oocyte maturation and fertilization. In vitrified or fresh oocytes, removal of CC represents a decrease in maturation or fertilization rates. Therefore, co-culture conditions with these cells are reported to increase in IVM and IVF rates. However, it is still necessary to investigate the mechanisms of action of the CC in a co-culture system that could be involved in their protection against apoptotic events, and their secretion factors that increase their developmental potential.

\section{Acknowledgement}

The authors thank and dedicate this work to Dr. Yvonne Ducolomb.

\section{Conflict of Interest}

No conflict of interest.

\section{References}

1. Rall WF, Fahy GM (1985) Ice-free cryopreservation of mouse embryos at $-196^{\circ} \mathrm{C}$ by vitrification. Nature 313(6003): 573-575.

2. Bajo J (2009) Fundamentals of Human Reproduction. Pan American Medical Editorial. Madrid Spain: 269-280.

3. Rojas C, Palolo JM, Albarracin JL, Mogas T (2004) Vitrification of immature pig oocytes: Study of distribution of chromosomes, microtubules and actin microfilaments. Cryobiology 49(3): 211-220.

4. Jin H Song, W Xin, Z Dai, S Chen, Z Sun (2012) Effects of cumulus cells on vitreous cryopreservation of human mature oocytes and clinical pregnancy outcomes. Rep Scien 19(2): 216-220.

5. Tharasanit T, Colleoni S, Galli C, Colenbrander B, Stout T (2009) Protective effects of the cumulus-corona radiata complex during vitrification of horse oocytes. Reproduction 137(3): 391-401.

6. Casillas F, Teteltitla Silvestre M, Ducolomb Y, Lemus AE, Salazar S, et al. (2014) Co-culture with granulosa cells improve the in vitro maturation ability of porcine immature oocytes vitrified with cryolock. Cryobiology 69(2): 299-304.

7. Wood MJ, Barros C, Candy C, Carrol CJ, Melendez J, et al. (1993) High rates of survivals and fertilization of mouse and hamster oocytes after vitrification in dimethylsulphoxide. Biol Reprod 49(3): 489-495.

8. McEvoy TG, Coull GD, Broadbent PJ, Hutchinson JS, Speake BK (2000) Fatty acid composition of lipids in immature cattle, pig and sheep oocytes with intact zona pellucida. J Reprod Fertil 118(1): 163-170.

9. Vieira AD, Messalina A, Barbieri DP, Lehmkuhl RC, Rubin MI, et al. (2002) Calves born after open pulled straw vitrification of immature bovine oocytes. Cryobiology 45(1): 91-94.

10. Fernández-Reyes F, Ducolomb Y, Romo S, Casas E, Salazar Z, et al. (2012) Viability, maturation and embryo development in vitro of vitrified immature and porcine oocytes. Cryobiology 64(3): 261-266.

11. Somfai T, Kikuchi K, Nagai T (2012) Factors affecting cryopreservation of porcine oocytes. J Reprod Dev 58(1): 17-24.
12. Vajta G (2000) Vitrification of the oocytes and embryos of domestic animals. Anim Rep Sci 60-61: 357-364.

13. Hochi S, Akiyama M, Minagawa G, Kimura K, Hanada A (2001) Effects of cooling and warming rates during vitrification on fertilization of in vitromatured bovine oocytes. Cryobiology 42(1): 69-73.

14. Betancourt M, Bonilla E, Casas E, Ducolomb Y (2003) Gamete maturation and fertilization in mammals. In Cellular and Molecular Biology Jimenez, F Merchant H (eds) Prentice Hall, México, 683-702.

15. Downs S, Daniel S, Bornslaeger E, Hoppe P, Eppig J (1989) Maintenance of meiotic arrest in mouse oocytes by purines: modulation of cAMP levels and cAMP phosphodiesterase activity. Gam Res 23(3): 323-334.

16. Zhang M, Ouyang H, Xia G (2009) The signal pathway of gonadotrophinsinduced mammalian oocyte meiotic resumption. Mol. Hum Rep 15(7): 399-409.

17. Sánchez F, Smitz J (2012) Molecular control of oogenesis. Bioch Biop Acta 1822(12): 1896-1912.

18. Yanagimachi R (1994) Mammalian fertilization in the physiology of reproduction. Knobil E, y Neill JD (Edit) 2nd Ed. New York. 189-317.

19. Schroeder AC y, Epigg JJ (1984) The developmental capacity of mouse oocytes that matured spontaneously in vitro is normal. Dev Biol 102(2): 493-497.

20. Wongsrikeao P, Kaneshige Y, ooki R, Taniguchi M, Agung B, et al. (2005) Effect of the removal of cumulus cells on the nuclear maturation, fertilization and development of porcine oocytes. Reprod Domest Anim 40(2): 166-170.

21. Zhang X, Miao Y, Zhao J, Spate L, Bennett M, et al. (2010) Porcine oocytes denuded before maturation can develop to the blastocyst stage if provided a cumulous cell-derived co-culture system. J Anim Sci 88(8): 2604-2610.

22. Casillas F, Betancourt M, Cuello C, Ducolomb Y, López A, et al. (2018) An efficiency comparison of different in vitro fertilization methods: IVF, ICSI and PICSI for embryo development to the blastocyst stage from vitrified porcine immature oocytes. Porcine Health Manag 4: 16.

23. Yoshida M, Ishigaki K, Nagai T, Chikyu M, Pursel VG (1993) Glutathione concentration during maturation and after fertilization in pig oocytes: relevance to the ability of oocytes to form male pronucleus. Biol Reprod 49(1): 89-94.

24. De Matos DG, Furnus CC, Moses DF, Martinez AG, Matkovic M (1996) Stimulation of glutathione synthesis of in vitro matured bovine oocytes and its effect on embryo development and freezability. Mol Reprod Dev 45(4):451-470.

25. Luvoni GC, Keskintepe L, Brackett BG (1996) Improvement in bovine embryo production in vitro by glutathione-containing culture media. Mol Reprod Dev 43(4): 437-443.

26. Tatemoto H, Sakurai N, Muto N (2010) Protection of porcine oocytes against apoptotic cell death caused by oxidative stress during in vitro maturation: role of cumulus cells. Biol Reprod 63: 805-810.

27. Cihangir N, Gorkemli H, Ozdemir S, Atan M, Duman S (2010) Influence of cumulus cell coculture and cumulus-aided embryo transfer on embryonic development and pregnancy rates. J Turkish-German Gynecol. Assoc 11(3): 121- 126.

28. Quinn P, Margalit R (1996) Beneficial effects of coculture with cumulus cells on blastocyst formation in a prospective trial with supernumerary human embryo. J Assist Reprod Genet 13(1): 9-14. 